ÉGYPTE

monde arabe

\section{Égypte/Monde arabe}

4 | 2007

Figures de la santé en Égypte

\title{
Entre initiation au jeu international, pouvoir colonial et mémoire nationale : le Conseil Sanitaire d'Alexandrie, 1865-1938
}

\section{Sylvia Chiffoleau}

\section{(2) OpenEdition}

\section{Journals}

Édition électronique

URL : https://journals.openedition.org/ema/1756

DOI : 10.4000/ema.1756

ISSN : 2090-7273

Éditeur

CEDEJ - Centre d'études et de documentation économiques juridiques et sociales

Édition imprimée

Date de publication : 31 décembre 2007

Pagination : 55-74

ISBN : 2-6905838-43-4

ISSN : 1110-5097

Référence électronique

Sylvia Chiffoleau, « Entre initiation au jeu international, pouvoir colonial et mémoire nationale : le Conseil Sanitaire d'Alexandrie, 1865-1938», Égypte/Monde arabe [En ligne], 4 | 2007, mis en ligne le 31 décembre 2008, consulté le 07 juillet 2022. URL : http://journals.openedition.org/ema/1756 ; DOI : https://doi.org/10.4000/ema.1756 


\title{
ENTRE INITIATION AU JEU INTERNATIONAL, POUVOIR COLONIAL ET MÉMOIRE NATIONALE : LE CONSEIL SANITAIRE D'ALEXANDRIE
} (1865-1938)

\begin{abstract}
ans la seconde moitié du xix siècle, à l'heure où se met en place un premier dispositif coordonné au niveau international de lutte contre les épidémies, l'Égypte est perçue comme un atout majeur et reçoit de la communauté internationale naissante la mission d'assurer en avant-poste la protection de l'Europe. L'Égypte se situe en effet à la charnière de trois continents, et transitent par le canal de Suez, non seulement une grande partie de la navigation mondiale, mais également d'importants flux de pèlerins musulmans, désignés à partir de 1865 comme les principaux responsables de la diffusion des épidémies. L'Égypte a été par ailleurs, depuis les années 1820, le théâtre d'une expérience originale et exemplaire de transfert de la médecine moderne ; sur son sol se croisent et travaillent de concert des médecins de toutes origines qui jouent un rôle majeur d'interface entre Orient et Occident. Le pays abrite enfin une institution, le Conseil sanitaire d'Alexandrie, née antérieurement à la mise en place de la coordination internationale mais que celle-ci va très largement mobiliser. En suivant l'évolution de son statut par rapport à la communauté internationale, en l'accompagnant dans sa pratique ordinaire et d'urgence, en examinant ses rapports aux puissances et aux institutions internationales, on dévoilera ici une institution tiraillée entre les attirances internationales, les contraintes coloniales et les aspirations nationalistes, mais qui a contribué très fortement à la genèse d'une santé publique internationale.
\end{abstract}

\section{LES TRIBULATIONS INTERNATIONALES D'UNE INSTITUTION SANITAIRE}

C'est bien sous le signe $d^{\prime}$ une volonté de coopération internationale qu'est né le Conseil sanitaire d'Alexandrie. Intuitivement convaincu du caractère 
contagieux des maladies épidémiques, et constatant l'efficacité du système empirique de protection mis en place par les nations européennes depuis le $x v^{\mathrm{e}}$ siècle, Muhammad 'Alî, confronté en 1831 à l'invasion du choléra, invite « de son propre mouvement " (Note de Muhammad 'Alî aux consuls européens en date du 8 octobre 1831, cité par Bérard, 1897, p. 3) les consuls des puissances européennes à se réunir en Intendance de santé publique destinée, d'une part, à le conseiller sur la politique de protection sanitaire du pays et, d'autre part, à fonder et gérer les établissements quarantenaires, notamment le lazaret d'Alexandrie. La démarche du vice-roi s'inscrit résolument dans une option quarantenaire qui commence à connaître alors de sérieuses remises en cause en Europe et relève chez lui d'un choix délibéré de recourir de façon pragmatique aux compétences étrangères pour faire avancer l'Égypte sur le chemin de la modernisation. Le souci de l'efficacité prime alors sur les considérations liées à la souveraineté. Pourtant, la pérennité de cette institution achoppe sur des problèmes internes qui rythmeront toute son existence : dissensions scientifiques, concurrence avec le service national de santé, luttes d'influence entre les nations représentées, partialité dans I'application des mesures quarantenaires... Lassé des querelles intestines, fort de ses succès militaires en Orient, Muhammad 'Alî supprime d'un trait de plume I'Intendance de santé en 1839, remplacée par une administration purement égyptienne. Le caractère international d'une telle institution ne va finalement pas de soi.

La situation particulière de l'Égypte ne saurait cependant exclure totalement l'élément étranger dans le champ de la santé, qui met de façon si évidente en jeu les intérêts commerciaux internationaux. En outre, l'Égypte abrite des communautés étrangères qui sont régies par le système des Capitulations ; en matière de santé publique comme en bien d'autres, les autorités égyptiennes ne peuvent agir directement auprès des membres de ces communautés. Dès 1843, Muhammad 'Alî doit de nouveau faire appel aux consuls qui obtiennent, au sein d'une nouvelle Intendance de santé, voix consultative uniquement en matière quarantenaire. C'est cette configuration de l'institution, quasi dépourvue de médecins et encore très égyptienne, dont prend connaissance, grâce à un long mémoire d'Henricy bey (Bérard, 1897, p. 14), la première conférence sanitaire internationale qui se réunit à Paris en 1851 et 1852 . Celle-ci recommande d'accentuer l'internationalisation de l'Intendance égyptienne, notamment en donnant voix délibérative aux délégués étrangers et en la dotant du droit de nommer les personnels, à l'image de ce qui se fait au Conseil de santé de Constantinople ${ }^{1}$. Le principe de la participation des consuls avec voix délibérative est finalement adopté en 1856 et se maintient, en principe uniquement en ce qui concerne les questions de quarantaine maritime et extérieure, après la fusion au sein d'une même Intendance générale sanitaire d'Égypte,

1. Dans un mouvement assez similaire à celui qui a pris place en Égypte, le Conseil de santé de Constantinople a été fondé par le Sultan en 1838. 
sise à Alexandrie, des deux services de santé, intérieur et quarantenaire, décidée par le gouvernement égyptien en 1858.

Dans la période qui suit, dominée par la personnalité du Dr Colucci, président de I'Intendance de 1860 à 1881, celle-ci se place dans un entredeux ambigu. Colucci obtient du vice-roi I'admission d'un nombre toujours croissant d'étrangers dans le Conseil, immixtion favorisée en outre par le désintérêt des fonctionnaires et des autorités égyptiennes pour les questions sanitaires ${ }^{2}$. Dans la pratique, le président gère les questions sanitaires en s'appuyant très largement sur les consuls et le vote de ceux-ci s'étend bien au-delà des seules questions quarantenaires (CADN, s.d.). Par ailleurs, I'Intendance veille soigneusement à placer ses réflexions et ses travaux sous I'autorité de la convention sanitaire de 1853, quelle que soit la faiblesse de celle-ci ${ }^{3}$, ce qui manifeste une volonté claire d'afficher une légitimité internationale. L'adhésion aux principes adoptés en 1853 se manifeste concrètement par le fait que le personnel médical supérieur en Égypte, les deux inspecteurs de Haute et Basse Égypte, tous les médecins de l'Intendance sanitaire et ceux des députations sanitaires, sont dès lors diplômés de facultés européennes (Procès-verbaux des séances du Conseil de l'Intendance générale sanitaire d'Égypte, 1886, p. 45). C'est d'ailleurs à cette époque que I'on prend I'habitude de désigner l'organe de décision des services sanitaires égyptiens sous le nom de "Conseil international de l'Intendance », puis tout simplement «Conseil sanitaire international ».

De son côté, le gouvernement égyptien, qui n'a pas été convié à la conférence de Paris et n'a donc pas ratifié la convention, ne se sent nullement lié par des obligations internationales. Il ne manque pas de rappeler que «si des décrets en date postérieure à cette convention ont changé l'organisation des services sanitaires en Égypte, ces décrets ont été fait sur la propre volonté des vice-rois sans aucune convention »(ANE, 1882). Certes, mais il est vrai aussi que cette législation, discutée au sein de I'Intendance, a été largement influencée par les réflexions menées au sein des conférences internationales, et ce depuis la convention avortée de 1852, même si pendant longtemps elles ne reçoivent pas de sanction juridique. La Turquie manifeste alors la même répugnance à admettre le caractère international du Conseil de Constantinople, pourtant plus fermement marqué. En 1879, le consul français à Constantinople s'insurge de l'omission du terme « international » sur la couverture d'un rapport du Conseil de santé, ce mot étant, dit-il, " l'épouvante de tout représentant de l'autorité turque ; ils admettent la chose, mais ne veulent pas de l'étiquette »(CADN, 1879).

2. À cette époque, la professionnalisation est bien engagée, mais la santé publique elle-même est quelque peu négligée et ne fait pas vraiment partie des enjeux.

3. Issue des travaux de la conférence de Paris de 1851-1852, celle-ci n'a été ratifiée que par la France et la Sardaigne et n'a pas eu d'existence effective. 
L'année 1866 avait pourtant marqué un pas supplémentaire dans le sens de l'internationalisation des institutions sanitaires d'Alexandrie et de Constantinople. L'Égypte n'avait d'abord pas été invitée à la conférence qui siège dans la capitale ottomane de février à septembre 1866, mais à partir de la $7^{\mathrm{e}}$ séance, le 8 mars 1866, on compte la présence du Dr Salem bey, professeur de clinique et de pathologie à l'École de médecine du Caire et médecin personnel de la mère du vice-roi. Sa présence à Constantinople permet d'établir une liaison directe avec I'Intendance qui peut ainsi suivre, voire intervenir par la voix du Dr Salem, au sein des débats. Les conclusions de la conférence, qui n'aboutit cependant pas à une convention, marqueront très fortement de leur empreinte la pratique et les décisions ultérieures de l'Intendance sanitaire d'Égypte qui ne cessera d'ailleurs de s'y référer ( $c f$. infra).

La bonne volonté internationale manifestée par l'Intendance sanitaire d'Égypte sera récompensée lors de la conférence de Vienne, en 1874, où I'Égypte est pleinement représentée par deux délégués, les Dr Colucci et Régny. Cette conférence, qui n'aboutit pas non plus à une convention, marque un infléchissement dans la politique de lutte contre les épidémies. Elle conclut en effet à l'inutilité des quarantaines terrestres, mais ne se prononce pas sur les quarantaines maritimes dont l'Égypte est en principe la gardienne, et initie le principe, appelé à se généraliser, du remplacement de la quarantaine par la désinfection. Dès lors, le traitement sanitaire de l'Orient va se dissocier de celui réservé au reste du monde et l'Égypte aura bientôt à gérer un cadre sanitaire particulier où domine la question du pèlerinage à La Mecque, soumis à un traitement sanitaire plus sévère, et d'inspiration fortement quarantenaire, que celui réservé au reste de la navigation.

La mise en cause du principe des quarantaines, qui gagne alors du terrain, rencontre un écho très favorable chez les Anglais, acteurs dont I'influence est grandissante en Égypte. Le territoire de la santé devient d'ailleurs un des lieux privilégiés où s'exprime la concurrence entre la France et la Grande-Bretagne, d'autant que I'Intendance se trouve, à l'image du reste de l'Égypte, dans une situation financière déplorable à la fin des années 1870, ce qui la fragilise considérablement. L’histoire médicale égyptienne est très liée à la France, non seulement à travers la personnalité de Clot bey mais aussi à cause de cette puissance au sein de I'Intendance (cf. Moulin, 2002). En effet, dès 1847, la France avait obtenu le privilège de voir siéger au sein de I'Intendance, en plus du consul, délégué officiel, son médecin sanitaire à Alexandrie. Cette participation active de la France ${ }^{4}$, associée à son rôle moteur dans le jeu sanitaire international, a pu conduire certains à qualifier I'Intendance " d'œuvre française »(CADN, 1880). C'est bien sûr fort exagéré mais, quoi qu'il en soit, la prééminence de la France est remise en cause par l'admission, en 1876, au

4. La France est aussi la destination principale des missions de formation et possède de nombreuses institutions hospitalières en Égypte. Cf. Moisan, 1997. 
sein de I'Intendance, du médecin sanitaire de Grande-Bretagne. Dans cette arène, I'opposition entre la France et la Grande-Bretagne va prendre la tournure d'un partage entre deux options sanitaires différentes: la seconde va désormais y défendre des positions hostiles à la quarantaine, tout particulièrement à l'égard de ses propres navires, alors que la France, plus directement exposée au risque contagieux par sa bordure méditerranéenne, demeure plus longuement attachée au principe quarantenaire. À partir de 1892, elle se fera la gardienne scrupuleuse des conventions internationales. Bien que le caractère international de l'Intendance ne repose longtemps sur aucune base juridique, la France entend en défendre à tout prix le principe. En effet, à ses yeux, en raison des options sanitaires de la Grande-Bretagne, le renoncement au caractère international de l'Intendance et son transfert sous influence britannique menacerait la protection sanitaire de l'Europe.

Face à l'agitation des puissances, et dans un ultime sursaut, l'Égypte reprend la main. Le gouvernement convoque une commission chargée de proposer une réforme du service sanitaire. Ses conclusions aboutissent au règlement du 3 janvier 1881 qui consacre définitivement la séparation des deux branches du service de santé, d'une part un Conseil intérieur basé au Caire, d'autre part le service de la quarantaine internationale qui relève d'une institution nommée désormais Conseil Sanitaire, Maritime et Quarantenaire (CSMQ), sis à Alexandrie. Les deux services sont réorganisés.

À partir de 1882, I'intrusion du facteur colonial va brouiller I'action internationale au sein du CSMQ. Si son président, Hasan bey Mahmûd, assure encore quelque temps le maintien du Conseil dans le giron de l'Égypte, en lui récusant tout caractère international, le remplacement de celui-ci par un président britannique, Mieville, en 1884, le place directement sous l'influence anglaise qui s'accroît, en outre, en raison du poids des voix des fonctionnaires égyptiens, la plupart de nationalité anglaise. L'égyptianisation de 1881 bénéficie finalement à la puissance coloniale. Dans ce contexte, la logique internationale suit néanmoins son cours. En 1892, une nouvelle conférence sanitaire internationale se réunit à Venise pour discuter des mesures à prendre pour assurer la protection sanitaire de I'isthme de Suez, notamment lors du retour du pèlerinage à La Mecque. Le CSMQ est officiellement chargé de la surveillance et de l'exécution de cette tâche et il est réformé en conséquence. Le nombre des membres égyptiens (ie. " anglo-égyptiens ») est réduit de 9 à 4 . Un décret khédivial en date du 19 juin 1893 modifie le décret du 3 janvier 1881 en conformité avec la convention sanitaire de Venise de 1892 : pour la première fois, la vocation internationale du CSMQ se réfère à une loi internationale. C'est sous I'ombrelle internationale, souvent malmenée par les Britanniques qui envisagent à plusieurs reprises la disparition de l'institution, que celle-ci continuera à exercer « une surveillance permanente sur l'état sanitaire de l'Égypte et sur les provenances des pays étrangers »(Décret khédivial de 1893, article 3) jusqu'à sa disparition en 1938. 


\section{LE CONSEIL SANITAIRE D'ALEXANDRIE, MATRICE D'UN MILIEU MÉDICAL INTERNATIONAL}

La permanence à Alexandrie, pendant plus d'un siècle, d'une institution sanitaire au caractère international, certes disputé, a contribué à faire naître dans la ville littorale, et plus largement dans toute l'Égypte, un milieu médical cosmopolite. Si la vocation du Conseil d'Alexandrie à surveiller les provenances extérieures est très tôt fixée, les conditions matérielles de la réalisation d'une telle surveillance, notamment l'existence effective de lazarets, ne sont cependant réunies que très tardivement, au tournant du siècle, au moment où ces mesures ne concernent plus guère que les pèlerins. Le dispositif mis alors en place offre des opportunités d'emploi recherchées par les étrangers, y compris dans les postes subalternes, en raison notamment d'un niveau de traitement supérieur à celui du cadre égyptien (Zananiri, 1996, p. 75). Ainsi, lorsqu'en 1897 un poste de garde sanitaire à Suez est mis au concours, poste subalterne à caractère non médical, le Comité des Finances du CSMQ, chargé de procéder à une première sélection des candidats, peut retenir cinq noms sur l'ensemble des dossiers déposés, tous étrangers. Le choix final est soumis au vote du Conseil qui donne 11 voix sur 15 à un certain M. Tyler, sans doute un Anglais (CADN, 1897a).

Le service quarantenaire offre des postes médicaux permanents et temporaires. Les seconds sont recrutés au moment de ce qu'il est convenu d'appeler

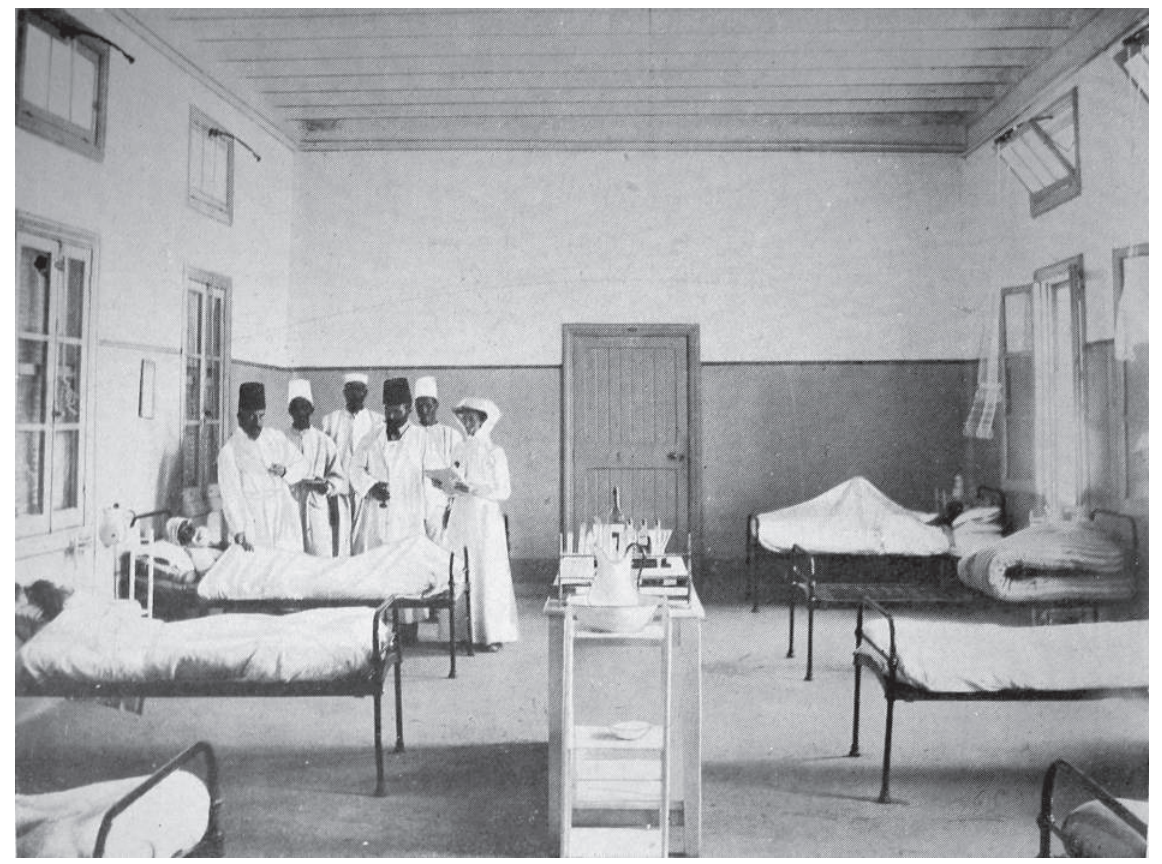

Salle d'hôpital du lazaret de Tor (in Dr René Briend, "Etablissements quarantenaires", Alexandrie, Société de publications égyptiennes, p. 60, s.d.) 
la «campagne quarantenaire », soit une période d'environ trois mois pendant lesquels le lazaret de Tor et les lazarets secondaires de la mer Rouge sont ouverts pour accueillir les pèlerins de retour du Hedjaz. Cinq médecins de pèlerinage sont ainsi appointés à Tor au tournant du siècle, sur la base d'un salaire décent de 20 LE par mois, ces places étant « en général briguées par des médecins séjournant habituellement en Égypte »(CADN, 1900a). De telles fonctions ne sont bien sûr pas sans danger et la campagne quarantenaire de 1900 a vu deux défections à Tor : le Dr Zeidan, victime d'une grave dysenterie dont il s'est cependant guéri, et le Dr Cladakis, décédé au campement d'une fièvre typhoïde (CADN, 1900b). Mais pour beaucoup, le recrutement temporaire au sein du service quarantenaire international représente une expérience exceptionnelle, faite de rencontres multiples et d'une confrontation directe à la foule souffrante, où l'on peut lire aussi les prémices d'un engagement humanitaire qui flirte avec des frissons d'aventure. Thérèse Barthas décrit avec enthousiasme son départ pour Tor en 1914, en compagnie d'une « colonie médicale des plus cosmopolite, Anglais, Français, Allemand, Autrichiens, Belges, Russes, Italiens, à côté de nos confrères d'Orient, sous l'étendard jaune des quarantaines marqué de l'étoile et du croissant d'Égypte ». Ensemble, ils vont « arrêter au passage tous les pèlerins qui reviennent de La Mecque et (...) nous ne les rendrons à la circulation que nets de tout microbe qui pourrait nuire à la santé du monde ». L'embarquement à Port-Saïd se pare pour elle d'accents romanesques : "C'est un conte à ravir un écolier avide d'aventures, que notre départ sur un bateau-hôpital, un bateau-mission, prêt, semble-t-il, pour quelque expédition fabuleuse » (Barthas, 1914, p. 85-86).

Le recrutement aux postes permanents ${ }^{5}$ représente un champ de concurrence forte entre les différentes nationalités représentées au sein du CSMQ : placer des médecins de sa nationalité entraîne un surcroît d'influence sur l'ensemble du système de santé et forme des relais de cette influence dans les provinces égyptiennes. Les tensions qui se nouent autour de la création de deux postes de doctoresse, en 1902, témoignent de l'importance de l'enjeu, ainsi que du processus de féminisation du service quarantenaire international. Arguant de la difficulté à recruter des doctoresses, le président du CSMQ, le Dr Armand Ruffer, nomme directement une Grecque à l'un des postes et s'apprête à disposer du second, destiné à l'office de Suez, pour y placer de la même manière, c'est-à-dire hors concours, une doctoresse anglaise. Face aux protestations du délégué français, le Conseil accepte à titre exceptionnel la nomination de la doctoresse grecque mais ouvre un concours pour le second poste. Quelques jours avant la clôture ce celui-ci, une candidature française, celle du Dr Marie Schültz, se présente face aux cinq candidates anglaises, dont celle soutenue

5. Le cadre supérieur demeure réservé aux étrangers, mais les postes médicaux de seconde et troisième classes sont ouverts aux Égyptiens et aux diplômés des Écoles de médecine d'Orient. 
par le Dr Ruffer. Le délégué de France au CSMQ et le consul de France entrent aussitôt en campagne auprès de leurs collègues pour défendre la candidate française, dont, assure le délégué français, les titres sont très supérieurs à ceux présentés par les autres concurrentes et qui est en outre recommandée par des personnages aussi prestigieux que les Dr Roux et Metchnikoff. De son côté, Ruffer bataille pour sa propre candidate, miss Vaugham. Alors en mission au campement de Tor, il envoie la veille du scrutin à I'Inspecteur général du CSMQ, président par intérim, instruction de donner « I'ordre aux délégués égyptiens de voter pour miss Vaugham ». Le vote s'établit à 8 voix pour chaque candidate puis, suite à une intervention directe de lord Cromer auprès des services consulaires des différentes puissances, sollicitée par Ruffer, miss Vaugham obtient, au cours d'un second scrutin, 10 voix contre 6 à Marie Schültz ${ }^{6}$. Certes Armand Ruffer a la réputation d'être un homme autoritaire, mais à ce trait de caractère s'ajoute l'importance des enjeux stratégiques : Suez est un lieu clé pour la navigation britannique et un relais anglais en un tel lieu permet de contrôler dans un sens favorable à celle-ci les mesures dictées par l'œuvre des conférences internationales que Ruffer, selon le délégué de France, considère « comme un ensemble suranné »(CADN, s.d.2). La résistance du fait colonial au processus international est ici encore évidente.

Les enjeux de concurrence entre les puissances sont plus vifs encore lorsqu'il s'agit de nommer un membre délégué du CSMQ et peuvent constituer parfois une mise à l'épreuve dans l'apprentissage des règles internationales. Tel est le cas en 1897. À cette date, le gouvernement anglo-égyptien vient de nommer un nouveau président, le Dr Ruffer, qui occupera cette fonction jusqu'en 1917, et s'apprête à remplacer l'Inspecteur général du service maritime et quarantenaire d'Égypte, alors le Dr Ardouin, en poste depuis 1881 et en âge de prendre sa retraite. L'Inspecteur général occupe le second rang dans la hiérarchie du Conseil et prend l'intérim du président en I'absence de celui-ci. Le poste d'Inspecteur relève du cadre égyptien, mais l'usage veut qu'il soit occupé par un médecin de nationalité française, usage que les Français souhaiteraient voir poursuivi. Or s'élève à ce propos une querelle d'interprétation de la convention de 1892. Le gouvernement égyptien entend en effet nommer directement un fonctionnaire de son choix à ce poste. La France proteste en affirmant que la convention de 1892, relayée par le décret khédivial de 1893, prévoit certes la nomination de ce poste par le ministère de I'Intérieur, mais sur désignation du Conseil. Les Anglais, qui entendent garder la main sur cette nomination, approuvent la lecture égyptienne de la convention et manœuvrent pour contourner le Conseil. Le gouvernement égyptien propose alors de résoudre le problème en retirant à I'Inspecteur la fonction de membre du CSMQ. Le siège vacant serait ainsi pourvu directement par les autorités égyptiennes. Mais une telle proposition, qui amoindrit le rôle de I'Inspecteur,

6. Celle-ci sera recrutée l'année suivante à l'office de Port-Saïd. 
impliquerait la révision de la convention sanitaire de Venise de 1892, ce que la France refuse, ainsi que les autres puissances consultées. Il est désormais plus important, aux yeux des Français et de la plupart des puissances européennes, de préserver l'intégrité des règlements internationaux que de défendre des positions nationales au sein du CSMQ. La crise dure des mois puis, suite à un accord direct et vraisemblablement confidentiel entre Proust et Ruffer (CADN, 1899a), le Dr Rist, un médecin français, est nommé directement par le gouvernement égyptien comme Inspecteur général, mais demeure membre du CSMQ. Le Dr Rist est d'ailleurs considéré avec suspicion par les Français, qui pensent qu'il ne « [leur] sera pas d'un grand secours pour la solution des questions où les intérêts français seront en cause »(CADN, 1899b). Une telle crise demeure cependant exceptionnelle et le plus souvent, il est admis que les personnels médicaux comme les membres du CSMQ sont remplacés par des personnes de même nationalité. L'accord se fait autour d'échanges de bons procédés et il existe même un « arrangement officieux fait avec les autres grandes puissances pour assurer le maintien des situations respectives actuelles et pour nous garantir contre des votes de surprise »(CADN, 1900c).

Le milieu médical né autour du CSMQ est très cosmopolite ; s'y croisent des médecins de toutes les nationalités européennes ainsi qu'un nombre important de médecins issus des écoles de médecine du Caire, Beyrouth et Constantinople. Certes le poids de la hiérarchie, qui place les Orientaux en position subalterne, ainsi que les rapports coloniaux, maintiennent une certaine distance entre médecins européens et orientaux, mais à Alexandrie même, ce milieu évolue dans des cercles et des salons où circulent l'information médicale, l'innovation, et aussi une conception des préoccupations sanitaires qui s'inscrit fortement dans I'international. Gaston Zananiri, qui fut longtemps secrétaire général du CSMQ, décrit dans ses mémoires ce milieu au croisement de la médecine et de la littérature. On y compte des femmes d'exception: Angélique Panayotatou, "bactériologue et femme de lettres », Thérèse Barthas, auteur de livres pour la jeunesse, dont l'époux est également fonctionnaire du CSMQ, Jeanne Puech-d'Alissac, connue sous le nom de plume de Jehan d'Ivray, elle-même épouse d'un médecin un temps en poste auprès du CSMQ (Zananiri, 1996, p. 76, 77). En outre, ce milieu est régulièrement visité, irrigué, par des individus qui occupent une position centrale et durable dans le champ médical international, notamment des Français. C'est le cas du diplomate Camille Barrère, un temps ministre de France en Égypte et qui a suivi, pendant près d'un quart de siècle, le cycle des conférences sanitaires internationales. Adrien Proust est en contact étroit avec le CSMQ et se rend luimême en Égypte en 1891. Certains médecins circulent sur l'ensemble du bassin méditerranéen, au gré des opportunités d'emploi. C'est par exemple le cas de Firmin Duguet, nommé en 1928 au poste d'Inspecteur général du CSMQ après avoir séjourné plusieurs années au Levant comme Inspecteur général des services d'hygiène des États sous mandat français et chargé par cette fonction de la surveillance sanitaire du pèlerinage à La Mecque. Une délégation égyptienne 
est envoyée chaque année dans le Hedjaz au moment du pèlerinage et à partir de 1865, I'Intendance compte un délégué ottoman. Des échanges permanents existent d'ailleurs entre le CSMQ et le Conseil de Constantinople, malgré la concurrence qui oppose ces deux institutions. L'ensemble de cette nébuleuse médicale et diplomatique forme, malgré les différences de statut et les tensions et concurrences de nationalités qui s'expriment souvent au sein du CSMQ, une certaine communauté de pensée et d'action.

\section{LE CONSEIL SANITAIRE D'ALEXANDRIE DANS LA PRATIQUE}

Si le milieu médical qui l'entoure est favorable à la circulation des idées, et probablement de l'innovation, le CSMQ lui-même ne se veut aucunement assimilé à une société savante. C'est une administration vouée à l'action concrète et réglementaire et les querelles scientifiques y sont d'autant plus bannies ouvertement que la première période de son existence, marquée par la querelle entre contagionnistes, autour de I'Intendance d'Alexandrie, et anti-contagionnistes réunis auprès de Clot bey au Caire, a laissé des souvenirs marquants de conflits stériles. Quoi qu'il en soit, à partir de 1865, un consensus assez général s'établit autour de la reconnaissance du caractère transmissible du choléra (P.V. des séances de l'intendance en 1865, p. 227), et $c^{\prime}$ est bien autour de cet accord que le CSMQ travaille à l'élaboration de la loi sanitaire égyptienne.

\section{Le travail réglementaire}

Antérieurement à 1865, et mises à part les toutes premières mesures quarantenaires décrétées par Muhammad 'Alî, la législation sanitaire égyptienne insiste sensiblement sur les aspects intérieurs, notamment les mesures d'hygiène publique et la mise en place d'un système civil de santé destiné principalement à répandre la vaccination antivariolique. En revanche, la législation quarantenaire demeure assez floue. Elle porte surtout sur la surveillance de l'état sanitaire des navires. Il est vrai qu'il n'existe alors qu'un seul lazaret, celui d'Alexandrie, le reste du littoral n'étant pourvu que de députations sanitaires au rôle essentiellement policier. Ce n'est qu'après la conférence de Constantinople en 1866, puis I'ouverture du canal de Suez en 1869, que I'effort législatif se porte sur la protection sanitaire aux frontières.

1866 marque une rupture profonde dans les modalités d'élaboration de la loi, laquelle s'articule dès lors aux prescriptions internationales alors même que celles-ci n'ont pas, pendant longtemps encore, force de loi. Cette annéelà, I'Intendance sanitaire est confrontée au difficile exercice qui consiste à élaborer sur le vif un règlement sur le retour du pèlerinage, menacé de choléra comme l'année précédente, au moment même où la conférence sanitaire 


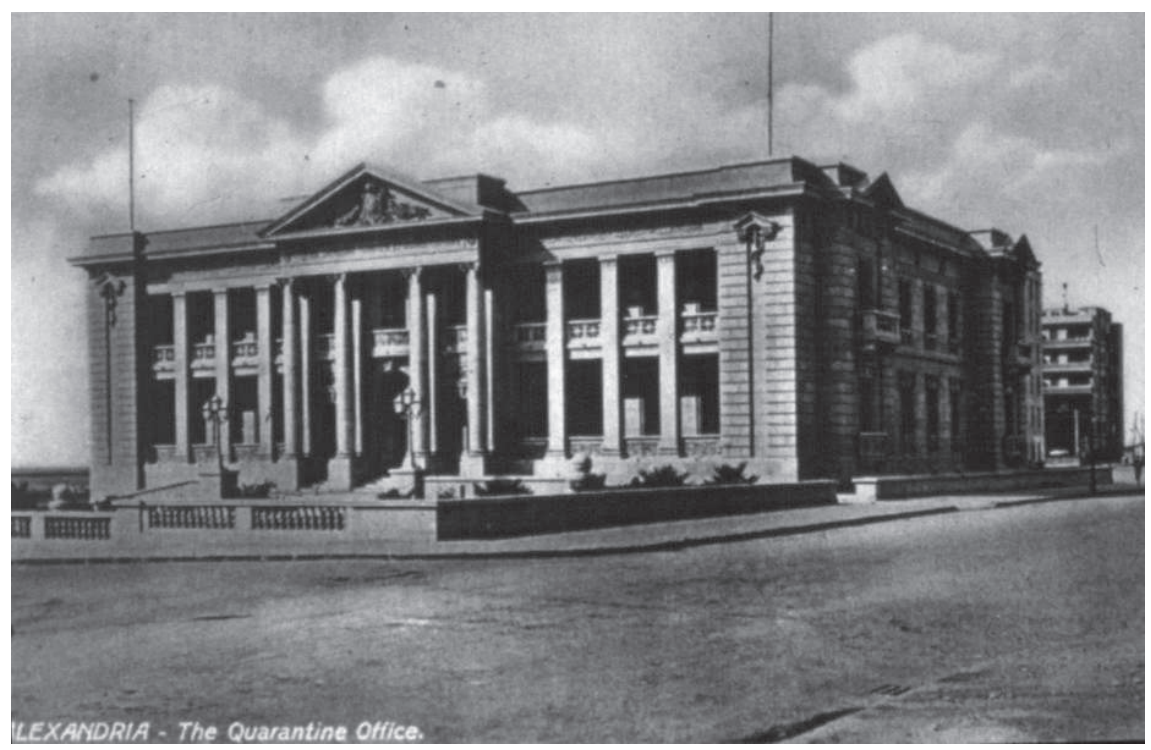

L'office de la quarantaine à Alexandrie

internationale poursuit ses travaux à Constantinople, et donc en tenant compte de ceux-ci, tout en s'ajustant aux réalités de terrain.

La gestion du choléra de 1865 par l'Intendance sanitaire d'Égypte a été violemment critiquée, notamment par la presse européenne. À l'époque, le poids des consuls, dont les options sont d'ailleurs appuyées par le président, le Dr Colucci, induit une vision très libérale des mesures de protection sanitaire. La priorité va aux intérêts commerciaux et les membres de l'Intendance manifestent une très grande répugnance à l'égard de toute mesure d'ordre quarantenaire qui pourrait entraver la marche de la navigation. Lorsque le choléra est signalé au Hedjaz en avril 1865, I'Intendance prend « aussitôt les mesures appropriées ", soit une quarantaine d'observation des caravanes à al-Wajh, sur la côte asiatique de la mer Rouge, qui n'excède pas 24 heures, accompagnée d'une visite médicale et suivie du transfert par train quarantenaire de Suez à Alexandrie où les pèlerins « sont conduits à bord des vapeurs pour être transportés dehors» (Ibid., p. XII). C'est à Alexandrie que le choléra se déclare tout d'abord. Les arrivages de Méditerranée, où le choléra s'est répandu ensuite, ne sont soumis qu'à une "visite médicale rigoureuse ». Quoi qu'il en soit, il n'existe alors que le lazaret d'Alexandrie, bien insuffisant pour recevoir tous les voyageurs et fort mal équipé pour satisfaire les classes supérieures de voyageurs. Ce n'est qu'à la fin du mois d'août, sous la pression internationale, que l'Intendance se résout à imposer des quarantaines, et encore sont-elle réduites dès le 13 septembre pour les voyageurs ordinaires en transit. Or la responsabilité des pèlerins dans la diffusion du choléra ayant été définitivement reconnue, I'Intendance va devoir adhérer, quoi qu'elle en ait, à un système quarantenaire plus sévère prescrit par la conférence. 
Ce sont précisément les critiques émises en 1865 qui incitent l'Intendance à placer ses réflexions sous l'égide de la conférence de Constantinople dès le début des travaux de celle-ci, en février 1866. Or se profile alors le retour du pèlerinage de 1866 et dans la crainte de ne pas recevoir à temps les prescriptions de la conférence, au cas où le choléra se déclarerait parmi les pèlerins, I'Intendance adopte des mesures à prendre, " sauf le cas où les dispositions du Congrès sur le même sujet arriveraient à temps » (Ibid., p. 127). Les navires chargés de pèlerins seront reçus aux Sources de Moïse, près de Suez, et les caravanes terrestres seront dirigées vers al-Wajh. Les pèlerins seront soumis en ces deux lieux à une visite médicale et seuls les malades seront gardés en quarantaine. La répugnance aux quarantaines demeure vive. Deux jours après cette décision, arrivent de Constantinople les consignes prescrites par la conférence au cas où le choléra se déclarerait parmi les pèlerins : toute communication maritime entre les ports arabiques et le littoral égyptien devrait alors être interrompue, ce qui impliquerait le stationnement, pour une durée indéterminée, de masses de pèlerins à Yambo et Djedda. Cette solution, très controversée, avait finalement été adoptée par la conférence au terme d'un long débat ${ }^{7}$. L'Intendance mesure immédiatement le caractère irréalisable et particulièrement périlleux pour les pèlerins de cette mesure. Mais le choléra ayant été signalé au Hedjaz, consigne est donnée aux compagnies de navigation d'interrompre les transports vers l'Égypte. Les pèlerins, abandonnés sans ressources dans les villes littorales du Hedjaz, parviennent néanmoins à s'embarquer de force ou, plus souvent semble-t-il, du gré des compagnies. Pour tenter de briser la chaîne de contagion, un vapeur égyptien est envoyé dans les eaux de la mer Rouge pour diriger les navires "délinquants » vers Tor, au sud de la péninsule du Sinaï, et les Sources de Moïse où les pèlerins doivent purger une quarantaine de 15 jours décidée par la conférence. L'Intendance doit ainsi, dans l'urgence et en contradiction avec ses propres principes, improviser des campements quarantenaires dans ces deux stations, premières ébauches de ce que seront les lazarets destinés aux pèlerins au tournant du siècle. La pratique de l'Intendance se plie donc aux prescriptions de la conférence, fort éloignées de celles qu'elle avait elle-même prévues. Ce faisant, elle manifeste son allégeance à l'autorité de la conférence et, au-delà, à la logique internationale. La même adhésion aux prescriptions internationales se poursuivra ensuite, en l'absence de toute contrainte juridique. De 1865 à 1892, le CSMQ joue ainsi un rôle majeur de relais de la législation sanitaire internationale en cours d'élaboration, et l'Égypte en forme un champ privilégié d'expérimentation.

Pour autant, la nature internationale du CSMQ, renforcée après la convention de 1892, n'est pas unanimement admise et continue à être contestée, notamment par l'autorité anglo-égyptienne. La répugnance du gouvernement

7. Les discussions sur cette question ont occupé pleinement les 6 premières séances de la conférence, du 23 février au 6 mars 1866. Cf. PV de la Conférence sanitaire internationale ouverte à Constantinople le 13 février 1866. 
égyptien à abandonner à une institution internationale hébergée sur son sol une part de la législation sanitaire se manifeste lors d'un conflit autour de la question du transit en quarantaine des pèlerins par le canal de Suez. Chaque question de ce type, concernant des procédures prenant place sur le sol égyptien, reçoit une réponse réglementaire ad hoc élaborée par le CSMQ. Ainsi, celui-ci avait voté un règlement sur le transit en quarantaine en 1890, mesures remises en vigueur en 1891, 1893 et 1895. Or cette année-là, le Conseil apprend que le gouvernement égyptien a édicté de son côté, en s'inspirant d'un rapport du Sanitary Department ${ }^{8}$, des « mesures de police à l'égard des navires à pèlerin transitant le canal maritime en état de quarantaine » dont certains aspects sont en contradiction avec le règlement du CSMQ. Un vif conflit s'élève alors entre les deux parties, le CSMQ protestant d'un empiètement inadmissible sur ses prérogatives reconnues par la communauté internationale, le gouvernement affirmant de son côté qu'il s'agit là de mesures de police, et non sanitaires. En 1897, afin d'éviter une nouvelle initiative de ce type de la part du gouvernement anglo-égyptien, le CSMQ décide de se placer une fois de plus sous l'ombrelle des conférences internationales et d'appliquer le règlement sur le retour du pèlerinage édicté à Venise, «à part quelques points techniques ou de juridiction qu'il est trop tard pour organiser »(CADN, 1897b), bien que la convention ne soit pas encore ratifiée. Dans le contexte colonial, le caractère international du CSMQ est soumis à des négociations permanentes, y compris après 1892 .

\section{L'engagement sur le terrain}

Outre son œuvre réglementaire, le CSMQ, ses équipes de médecins ${ }^{9}$ et ses personnels non médicaux, forment une société qui s'implique très concrètement, surtout à partir des années 1890, dans un travail de terrain au croisement des questions scientifiques et techniques. Leur patient travail va notamment aboutir à la réalisation du gigantesque lazaret de Tor, destiné aux pèlerins, considéré comme exemplaire, bien que son érection témoigne d'une chronologie décalée par rapport aux lazarets d'Europe : il n'atteint son plein fonctionnement qu'au moment où les autres lazarets disparaissent.

Si le principe de la mise en quarantaine des pèlerins est adopté dès 1865 , il faut attendre les années 1880 pour voir apparaître en Égypte une préoccupation plus marquée dans ce sens et des réalisations concrètes. La lacune principale tient au peu d'empressement manifesté par le gouvernement égyptien à financer des structures décidées en réunions internationales où il

8. Le service chargé de la gestion sanitaire intérieure, très étroitement aux mains des Anglais.

9. Les médecins du CSMQ réalisent aussi un travail proprement médical, comme en témoigne l'isolement du vibrion de Tor par Ruffer, en 1897. 
n’a, le plus souvent, pas été convié. La question des lazarets est néanmoins soulevée lors de la réorganisation des services sanitaires en 1881. Une commission est alors formée au sein du CSMQ, qui ne remet son rapport que trois ans plus tard. Celui-ci conclut à la nécessité de construire un lazaret de grande dimension à Alexandrie, pouvant accueillir 2000 personnes, un petit lazaret à Port-Saïd, destiné notamment aux pilotes accompagnant les navires transitant le canal en quarantaine, un autre à Tor pour les arrivages ordinaires du sud de la mer Rouge, prévu pour 200 personnes environ, et enfin un campement temporaire à al-Wajh pour les pèlerins revenant du Hedjaz (CADN, 1884). Seul Tor a véritablement vu le jour après un changement de vocation : c'est cette station qui accueillera finalement les pèlerins.

Le rôle du lazaret va bientôt s'étendre, avec la conquête des principes pastoriens, pour intégrer, au-delà de l'enfermement, la désinfection des bagages et des voyageurs. Pour équiper le lazaret en conséquence, une commission dite des améliorations est convoquée par le CSMQ. Son rapport, remis en mars 1891, préconise l'achat pour Tor d'une étuve supplémentaire, d'un type performant, en plus de celle s'y trouvant déjà, et la création d'un dépôt de médicaments et de substances désinfectantes à Alexandrie. Là encore, le principe de la désinfection est adopté en Égypte avant la convention de 1892 qui en fait une prescription internationale obligatoire. C'est d'ailleurs à la date de 1891 qu'est fixée dans ses grandes lignes la procédure quarantenaire qui aura cours à Tor pendant plusieurs décennies : visite médicale des arrivants, désinfection à l'étuve des vêtements et des bagages, douche collective pour les pèlerins, passage par les sections d'isolement, visite médicale quotidienne.

Ce système sera sans cesse amélioré par la suite, notamment en tenant compte d'un facteur qui prend de plus en plus de place, du moins dans les discours, celui du bien-être des pèlerins ${ }^{10}$. Chaque année les directeurs du campement de Tor remettent un long rapport au CSMQ où ils pointent les insuffisances de la station, lesquelles sont corrigées dans la limite des moyens disponibles. La conférence de Venise de 1897 ayant réclamé expressément la rénovation et la modernisation des lazarets de la mer Rouge, le CSMQ commence l'année suivante la construction à Tor d'un lazaret résolument moderne. En 1925, celui-ci comprend trois établissements de désinfection, des locaux pour les personnels, une poste, une station de télégraphe et téléphone, des bureaux pour les administrations de tutelle, des casernes de police et une place d'arme, quatre hôpitaux et une pharmacie ainsi que dix sections d'isolement qui, pour certaines, sont désormais formées de bâtiments et non plus de tentes (Zananiri, 1925). Les améliorations décidées se font selon les prescriptions internationales, mais leur réalisation se heurte sans cesse à l'insuffisance

10. Bien-être tout relatif comme en témoignent les récits de pèlerinage et la volonté constante de fuir l'épreuve du lazaret. Néanmoins, on ne manipule plus, au début du xx siècle, ces masses d'hommes avec la même légèreté qu'un demi-siècle auparavant. 
des financements qui reposent en partie sur la communauté internationale et principalement sur le gouvernement égyptien.

De façon générale, et malgré ces limites, les membres et personnels du CSMQ ne ménagent pas leur peine pour améliorer et humaniser le dispositif quarantenaire. Tel visite le quartier de l'armée d'occupation à Alexandrie pour examiner la qualité des tentes-ambulance utilisées par les troupes anglaises, fabriquées au Bengale, et qui pourraient avantageusement être utilisées à Tor ; tel autre teste et compare les machines à étuve, les produits de désinfection, les mérites des différents modèles de latrines (CADN, 1891). On s'informe de ce qui se fait à l'étranger (CADN, 1890). Les nombreux rapports produits par le CSMQ au cours de ces décennies, ainsi que les procès-verbaux de séance, fournissent un formidable témoignage des enjeux politiques autour du processus d'internationalisation, mais aussi des progrès scientifiques et des mesures concrètes subséquentes adoptées en Égypte, qui ont fait de ce pays un conservatoire en évolution d'un système quarantenaire obsolète partout ailleurs.

\section{La circulation de l'information}

Le CSMQ se présente comme un rouage essentiel dans I'apprentissage des procédures internationales en matière de santé. Plaque tournante de l'information sanitaire, il irrigue à son tour les centres de décision nationaux des puissances qui y sont représentées, et plus tard les instances sanitaires internationales.

Au départ, les consuls siégeant à l'Intendance sanitaire égyptienne semblent avoir bénéficié d'une large autonomie vis-à-vis de leurs administrations de tutelle. En 1865, le délégué italien propose d'introduire dans la pratique de I'Intendance l'inscription, sur l'invitation aux séances, des questions portées à l'ordre du jour afin que les délégués puissent en informer leur hiérarchie et en recevoir des instructions en retour. La proposition soulève bien des réticences, notamment de la part du président qui craint toujours des rumeurs trop promptes de diffusion de choléra et qui considère que cette délicate question doit être traitée « en famille » et sans la publicité que lui donnerait la diffusion d’un ordre du jour. Les délégués votent cependant en faveur d'une mise à l'essai de la proposition qui sera ensuite pérennisée $(P . V$. des séances de I'Intendance en 1865, p. 182). La logique internationale en gestation dans la pratique de l'Intendance impose d'en référer aux instances supérieures. Dès lors, s'instaure une routine de circulation de l'information, en dehors des situations d'urgence, du CSMQ vers les ministères européens des Affaires étrangères et retour. Le délégué transmet au consul de sa nation les procès-verbaux des séances du Conseil, éventuellement accompagnés d'autres documents ; le consul transmet l'ensemble au ministère des Affaires étrangères avec une note de synthèse soulignant les points principaux qui réclament éventuellement des instructions particulières. Le cas échéant, celles-ci suivent le même trajet en retour. La procédure s'effectue dans un délai de deux mois environ (CADN, 1898). 
Lorsque les débats du CSMQ soulèvent une question particulièrement délicate, il convient de dépasser le niveau diplomatique pour recourir à l'expertise scientifique. Dans le cas français, c'est le Comité consultatif d'Hygiène publique qui remplit ce rôle. À réception du courrier d'Alexandrie, le ministre des Affaires étrangères le fait suivre à son collègue du Commerce puis de I'Intérieur, ministères qui supervisent successivement les activités du Comité. Celui-ci est saisi et les problèmes soulevés sont débattus en séance. Le Comité renvoie son expertise par le même trajet diplomatique et son avis, c'est-à-dire bien souvent celui du Dr Adrien Proust, sert « de règle générale de conduite » (CADN, 1886) au délégué français au CSMQ.

Outre l'écho international qu'il renvoie de sa pratique ordinaire, le CSMQ sert de lieu de centralisation et de redistribution des bulletins épidémiologiques. Ceux-ci peuvent déclencher l'urgence en cas d'apparition, en quelque coin du globe, d'une maladie infectieuse épidémique. Dès 1874, I'infatigable Adrien Proust avait suggéré à la conférence de Vienne la création d'une Commission internationale permanente des épidémies en charge notamment de centraliser les informations. Faute d'aboutir concrètement, la proposition a suscité une évolution de la pratique tendant à améliorer la circulation des informations sanitaires. Dans les années 1880, le CSMQ reçoit, en plus des bulletins hebdomadaires des villes du Caire et d'Alexandrie, le relevé de la mortalité des ports de Calcutta, Colombo, Fiume, Hongkong, Karachi, Lisbonne, Londres, Macao, Malte, Odessa, Port-Natal et Trieste (Bérard, 1897, p. 59). Par ailleurs, les délégués sont tenus d'avertir sans retard leurs collègues en cas d'apparition d'une maladie épidémique sur le territoire dont ils sont originaires. Toutefois, le système est très imparfait et n'enregistrera de progrès notables qu'après l'apparition de la TSF. Longtemps la rumeur l'emporte comme mode de propagation de l'information, notamment en ce qui concerne les régions de la péninsule Arabique, pourtant particulièrement sensibles en raison du pèlerinage.

En gestation depuis un demi-siècle, l'idée d'une institution sanitaire internationale permanente aboutit finalement en 1907 avec la création de l'Office international d'hygiène publique (OIHP). Dès lors, la circulation de l'information est rationalisée et centralisée par celui-ci. Le CSMQ, qui devient en 1928 le bureau régional de I'OIHP pour le Proche-Orient, compte parmi les principaux relais du système mais cette transformation constitue aussi une étape décisive sur le chemin de sa liquidation.

\section{LA LIQUIDATION ET L'OUBLI}

L'impatience manifestée par le gouvernement égyptien à l'égard du CSMQ - les deux parties se disputant sans cesse les frontières de leurs prérogatives respectives - prend plus d'ampleur encore avec la montée en puissance du nationalisme. Les Égyptiens se trouvent d'ailleurs d'accord avec les Anglais pour contester l'existence de cette institution, mais pas pour les mêmes raisons. Les Britanniques y 
voient un organe obsolète qui entrave la liberté de la navigation. Les Égyptiens la perçoivent comme une structure internationale qui confisque une part de la souveraineté à laquelle ils aspirent de plus en plus. Dans le mouvement général du réformisme qui accompagne le nationalisme, l'Égypte va chercher à se substituer à cette instance internationale au nom d'une compétence désormais acquise.

L'offensive égyptienne est lancée lors de la conférence sanitaire internationale de Paris en 1903. Le délégué égyptien, Muhammad Chérif Pacha, propose de dissocier le rôle d'organe de diffusion de l'information sanitaire de celui de police sanitaire, le premier demeurant dans les prérogatives du CSMQ et le second revenant pleinement à l'Égypte. La proposition est appuyée par I'Angleterre mais farouchement contestée par la France. Influencée par le souvenir du choléra qui a ravagé l'Égypte l'année précédente, la conférence se range finalement à l'avis de la France et le maintien du statu quo est consacré par la convention sanitaire de 1903. Forts de I'indépendance partielle obtenue en 1922, les Égyptiens repartent à l'assaut de la conférence sanitaire internationale de 1926. La proposition égyptienne prévoit cette fois la prise de contrôle par le gouvernement du service vétérinaire, relevant jusqu'alors du CSMQ, et le renforcement de la représentation égyptienne au sein de celui-ci. La conférence cède sur les deux points bien que la diplomatie française y voit " une manœuvre claire pour préparer la disparition définitive de l'institution » (CADN, 1925).

La transformation du CSMQ en bureau régional de I'OIHP, en 1928, entraîne une redéfinition de l'institution qui tend à polariser son rôle d'organe de diffusion de l'information sanitaire vers les organismes internationaux alors que sa fonction de police sanitaire se place désormais dans un entre-deux qui laisse place à la négociation. Le second traité d'indépendance obtenu par I'Égypte en 1936 conduit ainsi à l'acte ultime de liquidation du CSMQ. Les Égyptiens le considèrent en effet comme une institution à caractère capitulaire ; il doit donc disparaître au même titre que tous les privilèges extraterritoriaux. C'est ce qui avait d'ailleurs été admis à Lausanne dans le cas du Conseil de santé de Constantinople. Après avoir hésité à imposer la suppression du conseil par mesure interne, l'Égypte fait finalement le choix d'une négociation internationale (CADN, 1939). Celle-ci se clôt lors d'une ultime conférence internationale qui se tient à Paris en octobre 1938 et qui accepte la dévolution des attributions du CSMQ à l'Égypte. L'Égypte s'engage à prendre en charge le fonctionnement du bureau régional de l'OIHP et à former, dans ce dessein, une commission comprenant des représentants techniques des pays ressortissants de ce bureau. C'est cette structure qui forme le noyau de ce qui deviendra plus tard le bureau régional de l'OMS.

Le processus politique qui a conduit à la disparition du CSMQ s'est accompagné, dans le champ scientifique, d'une délégitimation des préoccupations sanitaires qui sous-tendent les politiques mises en œuvre par le CSMQ. Durant la période de l'entre-deux guerres, le corps médical égyptien s'est attaché à substituer aux préoccupations sanitaires traditionnelles de l'Égypte, les 
épidémies, un intérêt prioritaire accordé aux maladies endémiques parasitaires, considérées comme "proprement égyptiennes»(Chiffoleau, 1997). Les travaux réalisés dans le cadre des deux congrès de médecine du Caire, en 1902 et 1928, témoignent de ce changement volontaire. Lors du premier congrès, qui réunissait exclusivement des médecins étrangers, deux sessions sont consacrées au choléra et à la peste. Le congrès de 1928, qui symbolise avec éclat la reprise en main du champ médical par les Égyptiens, fait presque totalement I'impasse sur les maladies épidémiques. De même, dans une somme sur l'histoire de la médecine égyptienne publiée sous l'autorité de 'Alî Ibrâhîm, l'un des ténors de la promotion des maladies parasitaires, seule une brève demi page, insistant sur le fait que l'Égypte vient de se débarrasser « totalement » de cette institution, est consacrée au Conseil sanitaire d'Alexandrie ('Alî Ibrahîm, 1939), lequel a pourtant largement contribué à diriger les destinés médicales de l'Égypte pendant plus de cent ans. L'Égypte nationaliste a entrepris une occultation délibérée de l'importance épidémiologique des épidémies et de I'œuvre du CSMQ.

En 1947, I'arrivée du choléra en Égypte secoue brutalement cette amnésie. Le département des épidémies, qui n'est désormais plus qu'un modeste service noyé dans l'organigramme du ministère de la Santé, doit se mobiliser dans I'urgence en imposant des mesures exceptionnellement sévères en matière de déplacement, d'isolement des malades et d'hygiène publique et individuelle. En outre, une vaccination générale est décrétée et c'est avec l'aide de la communauté internationale que le gouvernement organise la lutte, relayée sur le terrain par des volontaires de toutes origines. Les vaccins affluent de nombreux pays. Pour l'OMS, qui contrôle les procédures mises en place par les autorités égyptiennes et envoie sur place une délégation d'experts, il s'agit là de la première crise sanitaire à laquelle elle a dû faire face. La gestion du choléra de 1947 témoigne d'une organisation sanitaire internationale déjà performante, bien que sa structure définitive, l'OMS, ne soit née que très récemment, après une période de gestation d'un siècle. Dans ce long processus, le CSMQ a constitué un jalon essentiel et joué un rôle de premier plan en dépit des obstacles dressés par le pouvoir colonial puis les nationalistes égyptiens.

\section{RÉFÉRENCES BIBLIOGRAPHIQUES}

'Alî lbrahîm (dir.), 1939, Dalîl al-tibbî al-'âm li-misr wa-l-aqtâr al-charqiyya, (Guide médical général de l'Égypte et de l'Orient), Le Caire, F.E Noury et fils.

ANE (Archives nationales égyptiennes), 1882, Note du 8 avril, carton 4/2 (quarantaines).

BÉRARD, 1897, Le Conseil Sanitaire, Maritime et Quarantenaire d'Égypte, Alexandrie, Typo-lithogaphie V. Penasson.

BARThAS Thérèse, 1914, "Le retour de La Mecque. El Tor», La Revue de Paris, $\mathrm{n}^{\circ} 5,1^{\mathrm{er}}$ mars, p. 85-118. 
CADN (Centre des archives diplomatiques de Nantes), s.d., Résumé chronologique de l'histoire de l'Intendance sanitaire en Égypte, fonds Unions Internationales (fds UI), $1^{\text {er }}$ versement, série $\mathrm{S}$ " police sanitaire », carton 502.

- s.d. 2, Rapport du consul de France sur l'élection d'une doctoresse à Suez, fds UI, carton 511.

- 1879, Lettre du consul de France à Constantinople au ministre des Affaires étrangères, 6 août, fds UI, carton 491.

- 1880, Rapport du Comité consultatif d'Hygiène publique, A. Fauvel rapporteur, 28 juin, fds UI, carton 502.

- 1884, Rapport de la commission des lazarets au CSMQ, 4 mars, fds UI, carton 503.

- 1886, Lettre du ministère du Commerce au ministre des Affaires étrangères, 30 novembre, fds UI, carton 504.

- 1890, Fascicule sur le système quarantenaire de la Louisiane, fds UI, carton 538.

- 1891, Rapport présenté au CSMQ par la commission des améliorations, 26 mars, fds UI, carton 503.

- 1897a, CSMQ, procès-verbal de la séance du 23 juin 1897, fds UI, carton 503.

- 1897b, Lettre du Dr. Legrand, délégué p.i. du CSMQ au consul de France à Alexandrie, 7 mai, fds UI, carton 503.

- 1898, Lettre du consulat de France à Alexandrie au ministre des Affaires étrangères, 20 juillet, fds UI, carton 510.

- 1899a, Lettre du consulat général de France en Égypte au ministre des Affaires étrangères, 23 mai, CADN, fds UI, carton 507.

- 1899b, Rapport du consulat de France à Alexandrie au ministre des Affaires étrangères, 31 mai, fds UI, carton 507.

- 1900a, Rapport d'Harismandy, consul de France suppléant à Alexandrie au ministre des Affaires étrangères, 9 janvier, fds UI, carton 510.

- 1900b, Dr. Zachariadis, Rapport au CSMQ du directeur de campement quarantenaire de Tor, $1^{\text {er }}$ septembre, fds UI, carton 510.

- 1900c, Télégramme chiffré du Caire au ministre des Affaires étrangères, 14 décembre, fds UI, carton 511.

- 1925, Lettre du ministre de France en Égypte au ministre des Affaires étrangères, 29 décembre, fds UI, carton 520.

- 1939, Note du département Afrique-Levant sur la réforme du statut quarantenaire de I'Égypte, 21 août, fds UI, carton 524.

Chiffoleau Sylvia, 1997, Médecine et médecins en Égypte. Construction d'une identité professionnelle et projet médical, Paris, I'Harmattan/Lyon, Maison de l'Orient méditerranéen.

Molsan Dorothée, 1997, Médecine et médecins français en Égypte (18821914), mémoire de maîtrise d'histoire sous la direction du Pr. D. Chevallier, Université de Paris IV-Sorbonne, juin. 
Moulın Anne Marie, 2002, "L'esprit et la lettre de la modernité égyptienne. L'enseignement médical de Clot Bey » dans D. Panzac et A. Raymond, La France et l'Égypte à l'époque des vices-rois 1805-1882, IFAO, Cahier des Annales islamologiques, 22, p. 119 à 134.

Procès-verbaux des séances du Conseil de l'Intendance générale sanitaire d'Égypte, présidé par M. le Dr Antoine Colucci bey, depuis I'apparition, en Égypte, du choléra en 1865 jusqu'à sa disparition en 1866, Paris, Typographie et lithographie de Renou et Maulde, 1886.

PV de la Conférence sanitaire internationale ouverte à Constantinople le 13 février 1866, Constantinople, Imprimerie centrale, 1866.

ZANANIRI Gaston, 1925, Tor historique et quarantenaire, Alexandrie, CSMQ.

- 1996, Entre mer et désert. Mémoires, Rome, Instituto Storico Domenicano / Paris, Éditions du Cerf. 